\title{
Minimum Energy Coding for Wireless NanoSensor Networks
}

\author{
Murat Kocaoglu Ozgur B. Akan \\ Next-generation and Wireless Communications Laboratory \\ Department of Electrical and Electronics Engineering \\ Koc University, Istanbul, Turkey \\ Email:\{mkocaoglu,akan\}@ku.edu.tr
}

\begin{abstract}
Wireless nanosensor networks (WNSNs), which are collections of nanosensors with communication units, can be used for sensing and data collection with extremely high resolution and low power consumption for various applications. In order to realize WNSNs, it is essential to develop energyefficient communication techniques, since nanonodes are severely energy-constrained. In this paper, a novel minimum energy coding scheme (MEC) is proposed to achieve energy-efficiency in WNSNs. Unlike the existing minimum energy codes, MEC maintains the desired Hamming distance, while minimizing energy, in order to provide reliability. It is analytically shown that, with MEC, codewords can be decoded perfectly for large code distance, if source set cardinality, $M$ is less than inverse of symbol error probability, $1 / p_{s}$. Performance analysis shows that MEC outperforms popular codes such as Hamming, ReedSolomon and Golay in average energy per codeword sense.
\end{abstract}

\section{INTRODUCTION}

$\mathbf{N}$ ANO communications is a developing research area aiming the design of novel communication techniques for future nanodevices. Wireless nanosensor networks (WNSNs) are collections of nanosensors with communication capabilities. If successfully implemented, WNSNs are believed to have revolutionary effects on our daily lives [1]. Development of novel communication techniques suitable for nanodevice characteristics is essential for WNSN designs. In this paper, we study on nano-electromagnetic communications (nano-EM ), which uses EM waves as information carriers. In order to address novel characteristics of nanodevices, development of new communication techniques is required.

Nanosensors are devices of nano dimensions with accurate sensing capabilities. Potential nanosensors have significantly different characteristics and performance metrics than the macro nodes. With their extremely small size, nanosensor nodes can only provide limited energy. Hence, power and energy efficiency are of the most critical measures, and it is essential to develop energy-efficient communication techniques.

Carbon nanotubes (CNTs), which are the most promising building blocks for future nanodevices, can be efficiently used as nanoantennas. Resonant frequency of CNTs are shown to be

This work was supported in part by the Turkish Scientific and Technical Research Council under grant \#109E257, by the Turkish National Academy of Sciences Distinguished Young Scientist Award Program (TUBA-GEBIP), by IBM through IBM Faculty Award, and by Turk Telekom under Grant Number 11315-04 in the terahertz band. The main challenge of this band is the strong absorption of EM waves by water vapour molecules. The only practical way to use $\mathrm{THz}$ channel reliably is to use frequency windows scattered through the $\mathrm{THz}$ band, where absorption and molecular noise are low. Motivated with allowable frequency windows, in this work, we assume picoseconds long sinusoidal pulses as information carriers, which span a frequency band of $100-200 \mathrm{GHz}$, corresponding to the width of most of the frequency windows in the $\mathrm{THz}$ channel [2].

Wireless nanosensor networks (WNSNs) can be used for sensing and data collection with extremely high resolution and low power consumption for various applications [1]. There exists considerable research effort on WNSNs in the current literature. In [3], authors introduce carbon nanotube sensor networks and major challenges to be addressed for their realization. Authors in [1] provide a detailed survey on the state-of-the-art in nanosensors and emphasize potential applications and design challenges of WNSNs. In [2], THz channel absorption and noise characteristics are investigated and a new modulation scheme with 0.1 picoseconds long pulses as information carriers is introduced. In addition, authors demonstrate the potential of nano communications by investigating $\mathrm{THz}$ channel capacity via simulations.

In order to achieve reliable communication with minimum energy consumption in the nanosensor nodes, coding scheme should be chosen accordingly. Energy efficient coding schemes are extensively studied in the literature. Main metric used by researchers to measure energy efficiency is $E_{b} / N_{0}$, where $E_{b}$ is energy per bit and $N_{0}$ is the noise power spectral density. Incorporation of the effects of noise is important to ensure the reliability of the code in classical communication scenarios. However, $E_{b} / N_{0}$ is not the proper metric to achieve the objective of minimizing energy that transmitter dissipates. Minimum energy coding is choosing codewords for each source outcome such that average codeword power is less than any other choice of codeword sets or any other codeword mapping. It is first proposed in [4], where on-off keying (OOK) modulation is used with low weight coding to reduce energy dissipation of sensor nodes. In [5], authors propose using codewords with maximum weight of 1 . Recently, authors in [6] showed that, with TS-OOK modulation and low weight channel codes, interference can be reduced for potential WNSNs. 
Feasible, energy-efficient nanonodes make the development of WNSNs possible in the near future. However, to the best of our knowledge, developing channel codes in order to achieve energy-efficient nano communications has not been addressed.

In order to address this need, we propose minimum energy codes with Hamming distance constraint (MEC) in this paper. A novel minimum energy coding, MEC, is introduced, which minimizes average codeword energy for systems using OOK as the modulation technique. Different from the existing studies on minimum energy coding, we develop channel codes that yield minimum energy consumption per codeword, while satisfying a desired minimum Hamming distance, in order to provide reliability.

The remainder of this paper is organized as follows: We develop MEC in Section II-A. Analytical expressions related to MEC performance are developed in Section II-B. In Section III, simulation results on MEC and its comparison with popular block codes are presented, with feasible rate that a nanosensor node can supply. In Section IV, concluding remarks are given.

\section{Minimum Energy CODing With Hamming DisTANCE CONSTRAINT}

In this section, we develop a novel minimum energy coding scheme for energy-efficient nano communications. We propose using channel codes with minimum average code weight. Such codes are equivalent to minimum energy codes for the systems employing OOK modulation.

For block codes, a codebook is defined as any selection of fixed length codewords, which are mapped to source symbols. For unique decodability, i.e., error-free recovery of source symbols at the destination, this mapping should be one-toone. Hamming weight of a codeword is defined as the number of non-zero entries in the codeword. As we deal with binary codes only in this study, Hamming weight is equivalent to the number of $1 \mathrm{~s}$ in the codeword. Additionally, Hamming distance between two codewords is defined as the number of bits that they differ in. In minimum distance decoding, which is the presumed decoding strategy for this work, the received n-tuple is mapped to the closest codeword in terms of Hamming distance. A code is said to be t-error correcting if minimum distance of the code is $2 t+1$. Codes with larger minimum distance are more reliable, since more error patterns can be corrected.

Codewords with lower weight results less energy dissipation, when transmission of 0 symbol requires less energy than the transmission of 1 symbol. OOK is an example of such modulation schemes in which transmission of 0 s require no energy. The idea of using low-weight channel codes together with OOK modulation to reduce energy consumption is first proposed in [4]. Authors show that, for a given codebook, sorting codewords in increasing code weight order and assigning source symbols in decreasing probability order, such that most probable source symbol is mapped to the codeword with smallest weight, yields optimum average code weight. Later, authors in [5] propose using codewords with maximum weight
1. Such a mapping corresponds to minimum energy coding, when all zero codeword is mapped to the most probable source outcome. Recently, authors in [6] propose using low-weight channel codes for nano communications not only to reduce energy, but also to mitigate interference among nanonodes. We develop minimum energy channel codes with minimum Hamming distance $d$ to guarantee reliability, for communications in WNSNs employing OOK modulation. Proposed minimum energy coding (MEC) minimizes the expected codeword weight, depending on the source probability distribution. First, we obtain the minimum average code weight, then, derive analytical results related with performance metrics of MEC.

\section{A. MEC and Minimum Average Code Weight}

In this study, no source coding mechanism is assumed in the WNSN nodes, for complexity considerations. As a result, each codeword has the same probability of occurrence as the source outcomes that they are mapped to. This brings a new problem into the picture: What is the codebook selection that minimizes average code weight for any input probability distribution? This problem can be emphasized as finding the weight enumerator that minimizes the expected codeword weight for a given input probability mass function. It is trivial that for no Hamming distance constraint on the codewords, i.e., $d=1$, assigning codewords of maximum weight 1 yields minimum energy, as proposed in [5]. In order to obtain an analytical solution, we modify the minimum energy code problem such that code length $n$ is kept unconstrained. As a result, we obtain minimum expected code weight for codes with minimum distance $d$. The assumption of unconstrained $n$ makes the system realizable only for small size codebooks, i.e., if the nanosensor node is to transmit only a small set of events, since large delays are introduced with long codewords. However, this condition can be alleviated to some extend using multi-carrier modulation to divide codewords into parallel streams.

Let $M, d, p_{\max }$ represent number of codewords, minimum Hamming distance and maximum probability in any discrete probability distribution, respectively and $x$ be the source random variable. The following theorems give the limiting values for the expected code weight for MEC.

Theorem 1: Let $x_{i}$ be distributed with $p_{i} \in\left\{p_{1}, p_{2}, \ldots, p_{M}\right\}$ and $p_{\max }$ be $\max \left(p_{i}\right)$. For a desired minimum Hamming distance $d$, the minimum expected codeword weight, $E(w)$ is given by

$$
\min (E(w))= \begin{cases}\left(1-p_{\max }\right) d, & p_{\max }>\frac{1}{2}, \\ \frac{d}{2}, & p_{\max }<\frac{1}{2}, \text { if } d \text { even } \\ \left\lceil\frac{d}{2}\right\rceil-p_{\max }, & p_{\max }<\frac{1}{2}, \text { if } d \text { odd }\end{cases}
$$

Note that (1) is valid only for unlimited code length. Proof is omitted due to space requirements. The corresponding weight enumerator of the code is given as

$$
W_{\mathbb{C}}(z)= \begin{cases}z^{0}+(M-1) z^{d}, & p_{\max }>0.5 \\ z^{\left\lfloor\frac{d}{2}\right\rfloor}+(M-1) z^{\left\lceil\frac{d}{2}\right\rceil}, & p_{\max }<0.5 .\end{cases}
$$


A more realistic problem definition is as follows: What is the minimum expected code weight for code distance $d$ and maximum codeword weight $k$, where $k$ represents the maximum high symbols in a codeword that the nanonode can supply power for? This can be determined from the power budget of nanonode, transmission distance, atmospheric loss and required minimum SNR level at the receiver. If $k<\lceil d / 2\rceil$, there is no way to obtain a code with minimum distance $d$ and maximum code weight $k$. Hence, we assume that $k \geq\lceil d / 2\rceil$. It is clear that, if $p_{\max }<0.5$, bound given in Theorem 1 can be achieved for $k \geq\lceil d / 2\rceil$. However, if $p_{\max }>0.5$, for the case $\lceil d / 2\rceil \leq k<d$, it is easily seen that

$$
\begin{aligned}
\min (E(w)) & =p_{\max }(d-k)+\left(1-p_{\max }\right) k \\
& =p_{\max }(d-2 k)+k .
\end{aligned}
$$

Therefore, combining both cases yields the following.

Theorem 2: Let $x_{i}$ be distributed with $p_{i} \in\left\{p_{1}, p_{2}, \ldots, p_{M}\right\}$ and $p_{\max }$ be $\max \left(p_{i}\right)$. For a desired minimum Hamming distance $d$ and maximum codeword weight $k$, minimum expected codeword weight, $E(w)$ is given by

$$
\min (E(w))= \begin{cases}p_{\max }(d-2 k)+k, & p_{\max }>\frac{1}{2}, \\ \frac{d}{2}, & p_{\max }<\frac{1}{2}, \text { if } d \text { even }, \\ \left\lceil\frac{d}{2}\right\rceil-p_{\max }, & p_{\max }<\frac{1}{2}, \text { if } d \text { odd }\end{cases}
$$

for $\lceil d / 2\rceil \leq k<d$.

The corresponding weight enumerator is given as

$$
W_{\mathbb{C}}(z)= \begin{cases}z^{d-k}+(M-1) z^{k}, & p_{\max }>0.5 \\ z^{\left\lfloor\frac{d}{2}\right\rfloor}+(M-1) z^{\left\lceil\frac{d}{2}\right\rceil}, & p_{\max }<0.5 .\end{cases}
$$

Note that if we use all zero codeword to represent most likely source outcome (the case when $p_{\max }>0.5$ and $k \geq d$ ), we cannot distinguish if the transmitter sent data or remained silent, since both yield the same output unless some sort of synchronization between sender and transmitter exists. To provide reliability, we can put a minimum distance of $d$ with silence case also for all the codewords. This forces us to choose weight $-d$ codewords for all the input symbols to obtain the minimum expected code weight, which is $d$. However, we assume that all zero codeword can be distinguished from the silence case in the WNSN.

Note that MEC only provides the weight enumerator and minimum codeword length, not the actual codebook. Hence, minimum energy codes are not unique, since multiple codebooks satisfying the desired Hamming distance can be found.

\section{B. Analytical Results and Performance Parameters}

In this section, we analytically investigate MEC, that provides codes with the minimum average weight. These codes are equivalent to the ones with the minimum average codeword power, since OOK modulation scheme is utilized.

Let $p_{i}$ be the probability of event $i$ and $w_{i}$ represent weight of the corresponding codeword $c_{i}$. Power dissipated for $i^{\text {th }}$ event is represented as $P_{i}$ and given by $P_{i}=w_{i} P_{s y m}$, where
$P_{\text {sym }}$ is the symbol power. Then, the average transmission power of the nanonode is

$$
E(P)=\sum_{i=1}^{M} w_{i} p_{i} P_{\text {sym }}=E(w) P_{\text {sym }} .
$$

Note that (7) also represents the average transmission power per codeword and average transmission power per $\log (M)$ bits, since each codeword carries $\log (M)$ bits of information.

If the minimum Hamming distance between codewords is increased, more codeword errors can be corrected with minimum distance decoding. However, codeword length of MEC also increases with Hamming distance. Longer codewords result in a larger number of error patterns. Therefore, increasing minimum code distance does not necessarily increase reliability of MEC. Hence, analysis of error correcting capability of MEC for large Hamming distance is worth considering.

Let $d, n_{\text {min }}, p_{s}$ be Hamming distance, minimum required code length and probability of symbol error, respectively. Then, probability that transmitted codeword is correctly decoded (using the minimum distance decoding), is given by

$$
\xi_{d}=\sum_{i=0}^{\left\lfloor\frac{d-1}{2}\right\rfloor}\left(\begin{array}{c}
n_{m i n} \\
i
\end{array}\right) p_{s}^{i}\left(1-p_{s}\right)^{n_{m i n}-i} .
$$

In this paper, the codebook generation mechanism is such that codewords are chosen as shifted versions of each other by a minimal amount to satisfy Hamming distance constraint. Under this simple mechanism, minimum code length $n_{\min }$ required to have at least $M$ codewords with minimum Hamming distance of $d$, can be easily shown to be

$$
n_{\text {min }}=d+(M-2)\left\lceil\frac{d}{2}\right\rceil .
$$

We want to observe the limiting behaviour of probability of decoding the received codeword correctly as $d$ tends to infinity. Therefore, assuming very large and even $d$, without loss of generality, we obtain

$$
\begin{aligned}
\xi & =\lim _{d \rightarrow \infty} \sum_{i=0}^{\frac{d}{2}-1}\left(\begin{array}{c}
d M / 2 \\
i
\end{array}\right) p_{s}^{i}\left(1-p_{s}\right)^{d M / 2-i}, \\
& =\lim _{n \rightarrow \infty} \sum_{i=0}^{\frac{n}{M}-1}\left(\begin{array}{c}
n \\
i
\end{array}\right) p_{s}^{i}\left(1-p_{s}\right)^{n-i}, \\
& =\lim _{n \rightarrow \infty} 0.5\left(1-\operatorname{erf}\left(\frac{n / M-1-n p_{s}}{\sqrt{2 n p_{s}\left(1-p_{s}\right)}}\right)\right), \\
& = \begin{cases}1, & p_{s}<1 / M \\
0, & p_{s}>1 / M\end{cases}
\end{aligned}
$$

Expression in the limit in (12) is the cumulative distribution function of Gaussian distribution with mean $n p$ and variance $n p(1-p)$ and $\operatorname{erf}$ is the standard error function. Equality in (12) is due to the fact that, for large $n$, binomial distribution can be approximated by Gaussian distribution with aforementioned mean and variance. Note that error-free communication 


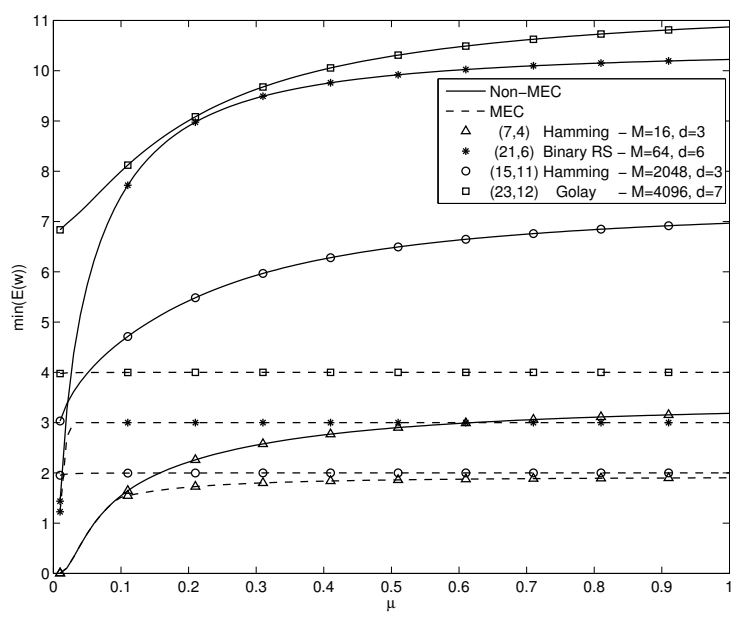

Fig. 1. Minimum code weight vs. source mean for $(7,4)$, $(15,11)$ Hamming $(21,6)$ Binary Reed-Solomon and $(23,12)$ Golay code and corresponding MEC

can be achieved, if source set cardinality is less than inverse of symbol error probability, by keeping Hamming distance sufficiently large. This result can also be interpreted as follows. If symbol error probability is decreased, analog reading of nanosensors can be quantized with smaller quantization steps (higher resolution).

\section{Performance Evaluation And Discussions}

In this section, we investigate error correction capability and energy-efficiency of MEC via simulations. MEC is compared with $(7,4),(15,11)$ Hamming, $(21,6)$ binary Reed-Solomon and $(23,12)$ Golay codes. As Hamming and Golay codes are compared with MEC in terms of average code weight and decoding probability, comparison with Reed-Solomon code is limited to expected code weight for the sake of simplicity and due to space requirements. Achievable rate by the nanosensor node is evaluated and shown to be currently limited by stateof-the-art power and energy limitations of CNT antennas and nano energy harvesting systems.

\section{A. Performance of Minimum Energy Coding}

1) Average code weight vs. source distribution: Performance of MEC in terms of expected code weight is compared with $(7,4),(15,11)$ Hamming, $(21,6)$ binary Reed Solomon and $(23,12)$ Golay codes in Fig. 1. Hamming codes are distance3 codes, and can correct 1 bit error whereas Golay code is distance-7 and can correct 3 bit errors. Minimum distance of $(21,6)$ binary Reed-Solomon code is known to be 6 . An $(\mathrm{n}, \mathrm{k})$ code maps $2^{k}$ sourcewords into channel codewords. For comparison, we use MEC with $M=2^{k}$. Expected minimum code weight is directly related with transmission energy per codeword. In order to reach minimum code weight for the Hamming, Reed-Solomon and Golay codes, more probable source symbols are assigned to codewords with less weight, using weight enumerators of these codes. Binary codes are chosen for comparison. Note that Reed-Solomon codes are non-binary codes, however, they can be expanded to binary codes as in [7]. Hence we have utilized binary expansion of 8 -ary $(7,2)$ Reed Solomon code for which a sample weight enumerator is given in [7]. We use normalized discrete samples of an exponential pdf with varying mean - $\mu$ in a fixed interval, to generate discrete distributions with different variances. It is clear from Fig. 1 that, MEC is superior, in other words, codes compared with MEC are not as efficient in terms of transmitted energy per codeword. Performance gap of the codes closes as mean, hence, variance of discrete distribution is decreased. This is intuitively expected. As $\mu$ decreases, $p_{\max }$ increases, since exponential pdf decays faster and contribution of the terms other than $p_{\max }$ to the expected weight diminishes. An interesting behaviour worth discussion is observed for comparison of MEC with $(7,4)$ Hamming and $(21,6)$ ReedSolomon codes. Before a critical $\mu$ value, Hamming and ReedSolomon codes operate very close to MEC performance. In this region, $p_{\max }>0.5$. Therefore, from (1), $p_{\max }$ is assigned to 0 -weight codeword and weights of the rest are uniformly distributed. Since Hamming and Reed-Solomon codes have a similar weight distribution with MEC in this case, they have very close $E(w)$ values. However, weight distribution for MEC changes abruptly for $p_{\max }<0.5$ which correspond to large $\mu$, surpassing the performance of other coding schemes.

2) Correct codeword decoding vs. symbol error: Codeword decoding performances of MEC, Golay and Hamming codes are illustrated in Fig. 2. Error correcting property of the proposed code is worse than the other codes considered. This is due to the vast difference in codeword lengths. Lengthy codes have more uncorrectable error patterns, which decreases the error correction probability for the same Hamming distance. It is observed that, as code distance is increased, probability of correct decoding tends to 1 , if symbol error probability, $p_{s}$, is less than inverse of source set cardinality, $1 / M$, and tends to 0 otherwise, verifying (13). This is intuitively expected, since increasing $M$ means increasing the amount of information to be transmitted, and this requires more reliable channels. Note that in our scenario, $M$ corresponds to the number of quantization levels at the nanosensor node.

\section{B. Achievable Rate of WNSN Nodes}

In this section, we investigate the feasibility of MEC for WNSN nodes, using state-of-the-art power and energy limits in nano-domain. It is theoretically calculated in [8] that a CNT antenna can radiate EM waves with power up to $5 \mu \mathrm{W}$. We take signal power at the CNT antenna constant as $1 \mu \mathrm{W}$. In [9], authors investigate an ultra-nano capacitor to store energy obtained from piezoelectric nano-generator energy harvesting system. Up to $800 \mathrm{pJ}$ of energy can be stored in the capacitor. Charging time for the capacitor depends on the frequency of vibration that nanonode is exposed to. In the linear region, in order to charge nano capacitor with $100 \mathrm{pJ}$ of energy, 160 cycles are required. If nodes gather energy from a $50 \mathrm{~Hz}$ source, such as a vent, 160 cycles correspond to a time of 3.2 seconds. Let energy harvesting system of nanosensor node 


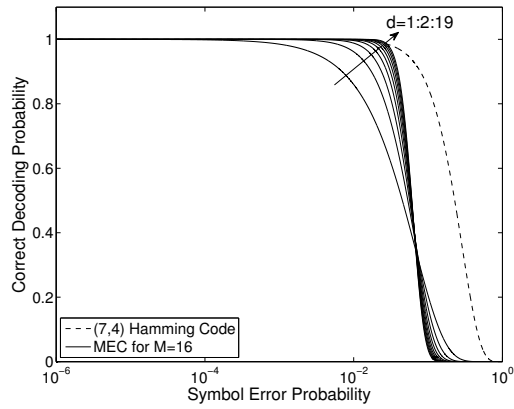

(a)

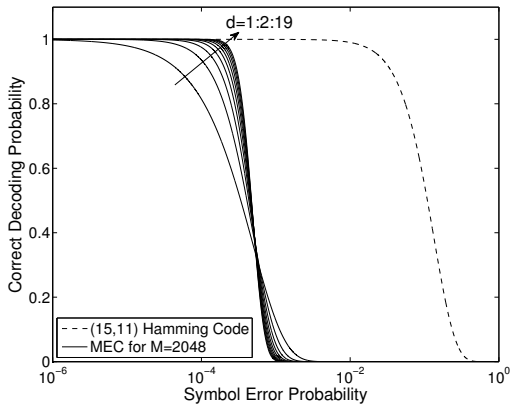

(b)

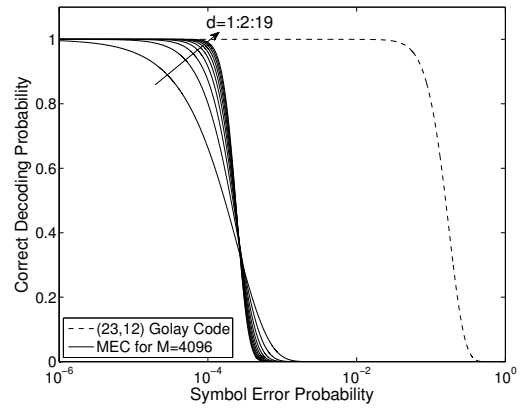

(c)

Fig. 2. Codeword decoding probability at the receiver for $(7,4),(15,11)$ Hamming and $(23,12)$ Golay codes and MEC with odd distances from 1 to 19 .

operate in this linear region. $T$, i.e., symbol time, is determined by channel characteristics, i.e., width of $\mathrm{THz}$ window and is around 10 picoseconds for a bandwidth of approximately 100 $\mathrm{GHz}$. Hence, symbol energy is constant and equal to

$$
\varepsilon_{\text {sym }}=P_{\text {sym }} T=10^{-5} \mathrm{pJ} .
$$

Therefore, a nanosensor node can transmit $10^{7}$ high symbols in 3.2 seconds. Finding the amount of information that can be carried with $10^{7}$ high symbols, we can calculate achievable bit transmission rate of a nanosensor node. Let $p_{\max }<0.5$ and $d$ be even for simplicity. Then, using (1) and (7), $\log (M)$ bits of information is carried with codewords of average energy $d \varepsilon_{\text {sym }} / 2$ on the average. Hence, average transmission rate is limited by

$$
R=\frac{2 \times 10^{7} \log (M)}{3.2 d}=6.25 \times \frac{\log (M)}{d} \text { Mbps } .
$$

It takes $n$ symbol times to transmit a single codeword. This sets another limit on the transmission rate, since

$$
R<\frac{\log (M)}{n_{\min } T}=\frac{\log (M)}{(d+(M-2)\lceil d / 2\rceil) T} .
$$

This bound is illustrated in Fig. 3. Comparing the results in Fig. 3 and (15), it is clearly observed that energy budget currently available at the nanonode limits its rate, rather than the codeword length. As a result, large codeword length, which is the major drawback of MEC, does not limit the available transmission rate. As illustrated in Fig. 3, code length allows transmission rates on the order of Gbps.

\section{CONCLUSIONS}

In this paper, we proposed a novel minimum energy coding scheme, MEC, for nano communications in WNSNs employing OOK modulation. Different from previous studies, MEC satisfies a minimum Hamming distance to guarantee reliability. It is analytically shown that, codewords can be decoded perfectly with MEC using large code distance, if number of quantization levels in WNSN node is less than inverse of symbol error probability. It is demonstrated via simulations that, the proposed code is superior to popular block codes such as Hamming, Reed-Solomon and Golay. The state-of-the-art

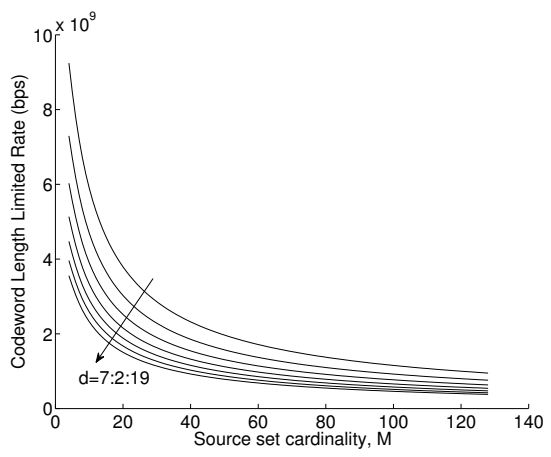

Fig. 3. Code length limited rate of nanonode in bps.

power and energy limits in nano domain are used to obtain achievable rates of WNSN nodes. Neglecting the processing power, nanosensor nodes are expected to transmit at data rates on the order of Mbps. Simulation results show that MEC can be used as an energy-efficient and reliable coding scheme in nano domain for future WNSNs.

\section{REFERENCES}

[1] I. F. Akyildiz and J. M. Jornet, "Electromagnetic Wireless Nanosensor Networks," Nano Comm. Net. (Elsevier), vol. 1, no. 1, pp. 3-19, 2010.

[2] J. M. Jornet and I. F. Akyildiz, "Channel Capacity of Electromagnetic Nanonetworks in the Terahertz Band," Proc. IEEE ICC 2010, Cape Town, South Africa, 2010.

[3] B. Atakan and O. B. Akan, "Carbon Nanotube Sensor Networks," Proc. IEEE NanoCom 09, San Francisco, CA, 2009.

[4] C. Erin and H. H. Asada, "Energy optimal codes for wireless communications," Proc. IEEE Conf. on Dec. \& Contr.", Phoenix, USA, 1999.

[5] Y. Prakash and S. K. S. Gupta, "Energy Efficient Source Coding and Modulation for Wireless Applications," Proc. Wireless Comm. \& Networking Conf., New Orleans, USA, pp. 212-217, 2003.

[6] J. M. Jornet and I. F. Akyildiz, "Low-weight channel coding for interference mitigation in electromagnetic nanonetworks in the terahertz band," Proc. of IEEE International Conference on Comm., ICC, 2011.

[7] F. Pollara, S. Arnold, "Binary Weight Distributions of Some ReedSolomon Codes," TDA Progress Report, vol. 42, no. 110, [Online], Available: http://ipnpr.jpl.nasa.gov/progress_report/42-110/110Q.PDF

[8] Y. Wang, Q. Wu, X.J. He, S.Q. Zhang and L.L. Zhuang, "Terahertz radiation from armchair carbon nanotube dipole antenna," Chinese Phys. $B$, vol. $18,2009$.

[9] J. M. Jornet and I. F. Akyildiz, "Joint Energy Harvesting and Communication Analysis for Perpetual Wireless NanoSensor Networks in the Terahertz Band," submitted for conference publication, 2011. 